\title{
Feedback control strategies for spatial navigation revealed by dynamic modelling of learning in the Morris water maze
}

\author{
Dirk Fey $•$ Sean Commins $\cdot$ Eric Bullinger
}

Received: 15 March 2010 / Revised: 10 June 2010 / Accepted: 9 August 2010 / Published online: 27 August 2010

(C) Springer Science+Business Media, LLC 2010

\begin{abstract}
The Morris water maze is an experimental procedure in which animals learn to escape swimming in a pool using environmental cues. Despite its success in neuroscience and psychology for studying spatial learning and memory, the exact mnemonic and navigational demands of the task are not well understood. Here, we provide a mathematical model of rat swimming dynamics on a behavioural level. The model consists of a random walk, a heading change and a feedback control component in which learning is reflected in parameter changes of the feedback mechanism. The simplicity of the model renders it accessible and useful for analysis of experiments in which swimming paths are recorded. Here, we used the model to analyse an experiment in which rats were trained to find the platform with either three or one extramaze cue. Results indicate that the 3-cues group employs stronger feedback relying only on the actual visual input, whereas the 1-cue group employs weaker feedback relying to some extent on memory. Because the model parameters are linked to neurological processes, identifying different parameter values suggests the activation of different neuronal pathways.
\end{abstract}

\section{Action Editor: Carson C. Chow}

D. Fey $(\bowtie) \cdot$ E. Bullinger

Systems and Modeling, Montefiore Institute,

University of Liege, 4000 Liege, Belgium

e-mail: fey@montefiore.ulg.ac.be

S. Commins

Department of Psychology, National University of Ireland,

Maynooth, Co. Kildare, Ireland
Keywords Autoregression • Dynamic modelling • Learning and memory $\cdot$ Random walk $\cdot$ Navigation • Spatial memory $\cdot$ Water maze $\cdot$ Autocorrelation • Autoregressive model

\section{Introduction}

The Morris water maze (Morris et al. 1982) is a standard test for studying spatial learning and memory. In order to escape swimming in the surrounding water, animals are required to locate a hidden platform (positioned just below the surface of the water). They do this by making use of the available cues in the environment. Although the task is widely used in neuroscience and experimental psychology to understand the behavioural and neural underpinnings of spatial learning, the exact mnemonic and navigational demands of the task are unclear. There is general acceptance that animals rely on distal cues to infer the platform location (often referred to as an allocentric learning), the exact strategy used depends on the number, location and availability of the cues (see Kealy et al. 2008 and references therein). Further, animals can adopt strategies that use cues in a beacon-like fashion or that are solely based on self movements and memorised motor actions (egocentric learning; see Moghaddam and Bures 1996). However, the dissociation of spatial from nonspatial strategies is not always possible (Harrison et al. 2006). It is therefore crucial for any study of spatial learning and memory, to understand the navigational demands of the tasks and the strategies solving it. The present study clarifies the navigational demands of the Morris water maze and quantifies the effectiveness of different strategies using a (data-driven) dynamic modelling 
approach. In contrast to other modelling studies in the field of neuroscience that utilise and examine complex networks of neurons (Burgess 2008), the approach presented here focuses on whole behaviour (Cain and Saucier 1996) and offers a simple model that is identifiable from experimental data of swimming paths.

Traditionally, rather crude performance measures are employed to assess learning, such as escape latencies or the time spend in different areas of the pool (Maei et al. 2009). In contrast, we provide a more comprehensive time series analysis of rat swimming dynamics and propose a mathematical model of navigation as a stochastic process. The basic idea is that prior to training, rats exhibit random movements and with learning these movements become increasingly goal oriented. Between two measuring points, a rat moves a certain distance (step size) in a certain direction (heading). This movement can be described by a directed random walk, whereby the step size and heading change are random processes.

The dynamic modelling approach offers full control over multiple variables and a much richer analysis compared to measuring only simple performance statistics. For example it allows testing whether a purely egocentric strategy would be sufficient to solve the task. Real animals in the watermaze may employ a variety of different strategies (even in a single trial) such as avoiding the pool border, approaching a cue, etc (Harvey et al. 2009), which makes it very difficult to assess the effectiveness of these individual strategies experimentally. Using a modelling approach, one can formally specify different strategies, and then analyse these in simulations, either individually or in combination. As such the effectiveness of different strategies and their relative contribution to the performance in terms of escape latencies can be assessed effectively.

\section{Experimental data}

The data was obtained by Harvey et al. (2009). Male Wistar rats (aged 3 months 250-350 g, Biomedical Facility, University College Dublin) were divided into two groups ( 1 cue and 3 cues). All animals $(n=16)$ were given 4 trials per day for 5 days to acquire the watermaze task. The Morris water maze consisted of a circular pool (1.7 m diameter). Rats could escape from swimming by locating a hidden platform $(9 \mathrm{~cm}$ diameter) located in all experiments in the middle of the northeast quadrant of the pool. The platform was submerged $2 \mathrm{~cm}$, rendering it invisible to the rats. The pool was surrounded by a black curtain located approximately $50 \mathrm{~cm}$ from the pool wall. Different numbers of cues suspended on the inside of the curtains were available for the different groups. The 3 cues group had two light bulbs (in the northwest and northeast corner), and a rectangular sheet of white paper $(55 \mathrm{~cm} \times 81 \mathrm{~cm}$, east side). The 1 cue group had a single light bulb located in the northeast corner. Collected data consists of recordings of rat swimming paths ( $\mathrm{x}-\mathrm{y}$ coordinates) in successive trials with neglectable positional error and a temporal resolution of $0.2 \mathrm{~s}$ over at most $60 \mathrm{~s} \mathrm{(after}$ which the trial classifies as unsuccessful), resulting in 50 to 300 data points per trial.

\section{Stochastic process modelling}

The dynamic model is conceptually based on a directed random walk (Fig. 1(a)) and a feedback loop of the heading change having a modular structure (Fig. 1(b)). The random walk is modelled as a discrete-time system:

$x_{t}=x_{t-\Delta t}+\Delta r_{t} \sin \left(\alpha_{t}\right)$,

$y_{t}=y_{t-\Delta t}+\Delta r_{t} \cos \left(\alpha_{t}\right)$,

$\alpha_{t}=\alpha_{t-\Delta t}+\Delta \alpha_{t}$,

where $x_{t}, y_{t}$ and $\alpha_{t}$ denote position and heading of the rat at time $t . \Delta t$ is the sampling time of the experimental data. The step size $\Delta r_{t}$ and the heading change $\Delta \alpha_{t}$ are random processes to be identified from the data. Based on our data analysis, we assume that the step size is an independent random variable (the crosscorrelation between step size and heading change shows a high pvalue of $>0.2$ and values $20 \times$ smaller than the autocorrelation of the heading change), whereas the heading change is a standard autoregression model extended by an input term $u$ :

$$
\Delta \alpha_{t}=\sum A_{i} \Delta \alpha_{t-i \Delta t}+u,
$$

where $A_{i}$ are coefficients describing the relative contributions of the past values on the current value. Two considerations motivated Eq. (2). First, rats do not change their heading completely randomly, as they tend to swim coherent curves. For example, when the rat turns left at one time instant, it tends to keep turning left for a certain amount of time, thus swimming a left curve. This effect can be modelled mathematically by making the change of heading dependent on previous heading changes, i.e. nonzero coefficients $A_{i}$. Second, rats are able to control their change of heading, which is mathematically modelled by the input term $u$ realising a feedback mechanism:

$u_{t}=K \hat{e}_{t}+v_{t}$. 
(a)

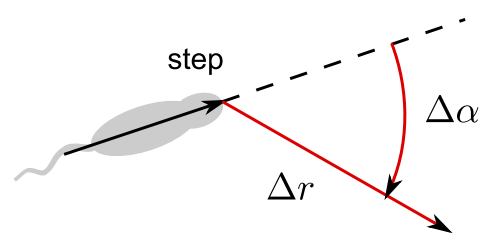

step +1 (c)

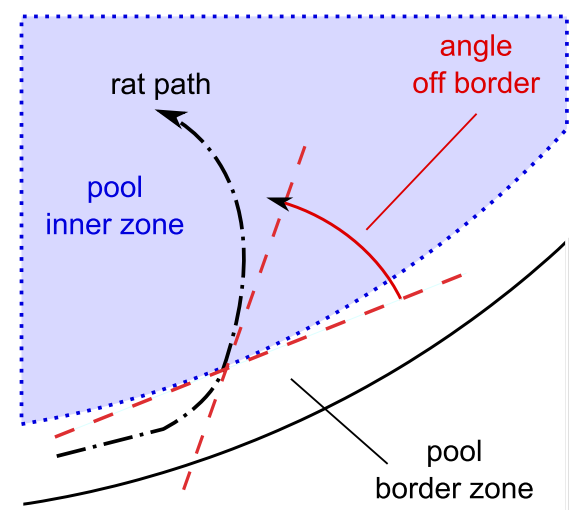

(b)

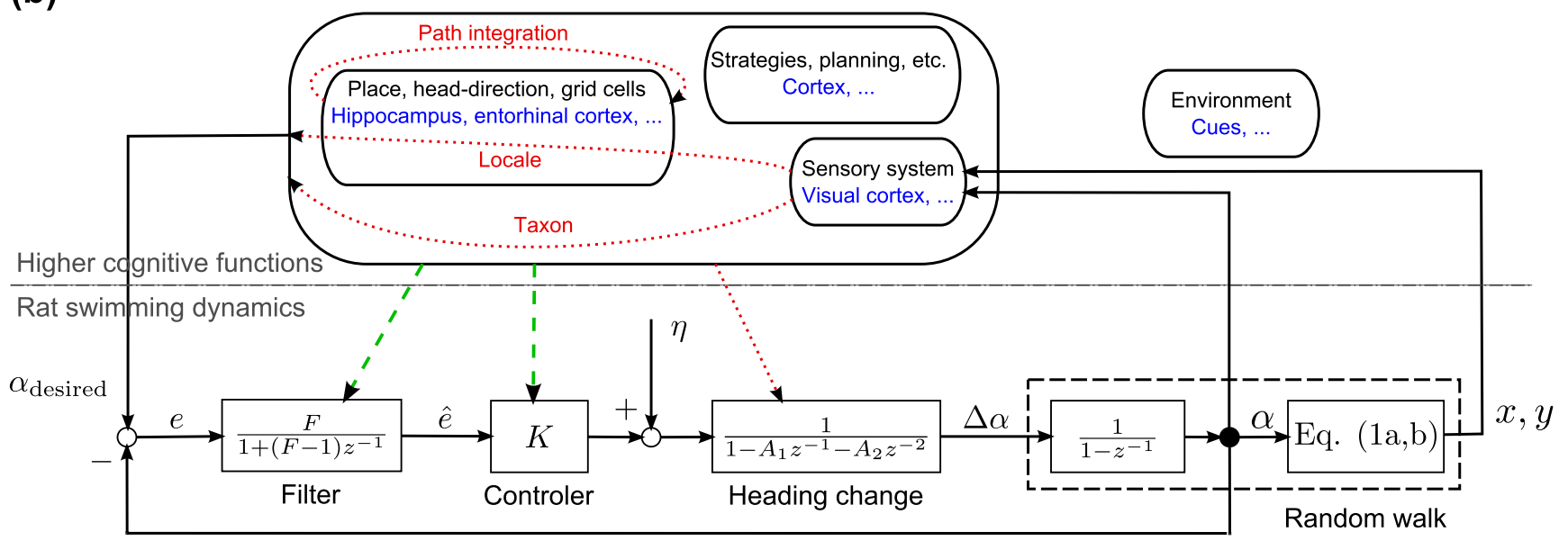

Fig. 1 Sketch of the model. (a) Illustration of the random walk with $\Delta r$ denoting the step size and $\Delta \alpha$ the heading change. (b) Overview of the heading change model (dynamics) and its relations to the neurophysiology (cognitive functions). On the path dynamics level, $x, y$ is the rat's location, $\alpha$ is the rat's actual heading, $\alpha_{\text {desired }}$ the rats desired heading, $e$ the heading error, $\hat{e}$ the rats estimate of the heading error, $u$ the input to the heading change model and $\eta$ a random variable with a normal distribution. The variable $z$ arises from the $\mathrm{z}$-Transform of the system, and can be understood as a time shift operator, i.e. $z^{-i} x(t)=x(t-i \Delta t)$. The neurological level comprised different brain regions and neuronal pathways and determines $\alpha_{\text {desired }}$ as well as the model parameters $F, K$ and $A_{i}$. (c) Illustration of the angle at which the rat leaves the pool border
Table 1 Overview of the model and the distributions identified from the data

\begin{tabular}{|c|c|c|c|}
\hline \multicolumn{2}{|c|}{$\begin{array}{l}\text { do } 300 \text { times } \\
\text { perform strategy } \\
\text { perform step according to Eq. (1) } \\
\text { if location = on-pool-border } \\
\text { perfom step along border } \\
\text { with probability p leave border } \\
\text { endif } \\
\text { if location = platform } \\
\text { terminate simulation } \\
\text { endif } \\
\text { enddo }\end{array}$} & \multicolumn{2}{|c|}{$\begin{array}{l}\text { case random } \\
\text { calculate } \Delta \alpha \text { according to Eq. (2) with } u=0 \\
\text { case cue-based } \\
\text { if nsteps }<1 \\
\text { choose a cue randomly as target } \\
\text { set nsteps }<=10 \text {, endif } \\
\text { calculate } \Delta \alpha \text { according to Eqs. (2), (3) \& (4) } \\
\text { case place-control } \\
\text { if location }=\text { target } \\
\text { choose new target according to Eq. (5), endif } \\
\text { calculate } \Delta \alpha \text { according to Eqs. (2), (3) \& (4) }\end{array}$} \\
\hline Process & Distribution & Probability density function & Parameters \\
\hline Step size & Rayleigh & $\frac{x}{b^{2}} \exp \left(\frac{-x^{2}}{2 b^{2}}\right)$ & $b=8$ \\
\hline Heading change & Normal & $\frac{1}{\sigma \sqrt{2 \pi}} \exp \left(\frac{-(x-\mu)^{2}}{2 \sigma^{2}}\right)$ & $\mu=0, \sigma=10$ \\
\hline Probability to leave border & Constant & & $p=1 / 8$ \\
\hline Angle off border & Log-normal & $\frac{1}{\sigma \sqrt{2 \pi}} \exp \left(\frac{-(\ln x-\mu)^{2}}{2 \sigma^{2}}\right)$ & $\mu=3.5, \sigma=0.78$ \\
\hline
\end{tabular}


Here, $\hat{e}_{t}$ is the rat's estimate of its heading error, $K$ is a proportional feedback gain and $v_{t}$ a normal distributed random number (Gaussian noise). The higher the gain, the faster the desired heading is achieved. We assume that the error estimate is realised as a low pass filter

$\hat{e}_{t}=(1-F) \hat{e}_{t-1}+F\left(\alpha_{\text {desired }}-\alpha_{t}\right)$,

where $0 \leq F \leq 1$ is a weighting factor. Loosely speaking, the higher the weighting factor, the more the rat trusts its visual input; the smaller the weighting factor, the more the rat trusts its memory. Using an error estimate rather than the actual error directly achieves a much better model fit to the data (Section 4) and is further interpreted in Section 5.

The above considerations mainly concern the rats swimming behaviour in the interior of the pool. Along the pool border, rats exhibit a distinct swimming behaviour termed thigmotaxis (Fig. 1(b)). The complete model captures both behaviours and is summarised in Table 1.

\section{Results}

This section identifies the mathematical model from experimental data and analyses different navigational strategies. Estimating all parameters from the recorded swimming paths is possible because the described model is simple and largely linear (except for Eq. (1)).

\subsection{Learning not reflected in the distribution,} but in the autocorrelation of the heading change

The probability density functions of the random variables were identified by analysing the recorded swimming paths using the MATLAB ${ }^{\circledR}$ Statistics Toolbox ${ }^{\mathrm{TM}}$ (Fig. 2(a)-(d)). The distributions of step size and heading change do not change significantly with training (Fig. 2(e), (f)). This is not surprising, because those variables do not contain positional information. However, the autocorrelation of the heading change, which describes how current heading changes correlate to past heading changes for one time step back (lag one, i.e. $0.2 \mathrm{~s}$ ), two time steps back (lag two, i.e. $0.4 \mathrm{~s}$ ) and so forth: $\sum_{i} \Delta \alpha_{i} \Delta \alpha_{i-\mathrm{lag}}$, increases over days, reflecting the rats learning progress (Fig. 2(g)).

\subsection{Open loop model mimics swimming behaviour of day one}

We identified the parameters $A_{i}$ of the heading change model in open loop using the data of day one, when we assume the rat's swimming behaviour is not directed towards a particular goal $\left(\alpha_{\text {desired }}=\alpha\right)$ and the input $u$ is Gaussian noise $\left(u_{t}=v_{t}\right)$. This renders Eq. (2) a simple autoregressive model that can be identified using the Yule-Walker equations. We found that a second order model (i.e. $A_{i}=0$ for $i>2$ ) explains the observed autocorrelation sufficiently well (Fig. 2(h)). Only slightly different coefficients were obtained for the 3-cues and 1-cue group. The simulated swimming paths and the resulting simulated escape latencies are in good accordance with those of the wet lab experiments.

\subsection{Closed loop model reveals different feedback mechanisms}

We identified the parameters $K$ and $F$ of controller and filter in closed loop using the data of day five, when the rats direct their heading using the described feedback mechanism. Here, the Yule-Walker equations are not applicable due to the feedback. Instead, we used simulations to minimise the least squares error of the autocorrelation of the heading change (Fig. 2(i)-(1)).

We found an inherent difference in the navigational control strategy depending on whether one or three cues were available. Despite the fact that learning occurs equally fast in both cases, 3-cues rats employ a stronger feedback $(K=0.42)$, compared to the 1-cue rats $(K=0.32)$. In addition, the 3 -cues group seem to rely only on the currently observed error, i.e. $F=1$, whereas the 1 -cue group rely to $24 \%$ on their memorised estimate, i.e. $F=0.76$. We repeated this analysis using different, more complex control models (data not shown). All gave similar results, showing higher, immediate control for 3-cue rats, and lower, delayed control for 1-cue rats.

\subsection{Model analysis assesses efficiency of navigational strategies}

A first simulation experiment implemented a purely egocentric strategy in which rats learn to avoid the border (platform located somewhere in the interior of the pool). Border avoidance is modelled by a change of the probability to leave the border. Simulations revealed that solely avoiding the border slightly decreases the escape latencies, but that this effect is rather minor (Fig. 3(a)).

A second simulation experiment implemented a cuebased egocentric strategy in which the rats approach different cues for a certain (random) amount of time. Unsurprisingly, the analysis of the resulting escape 

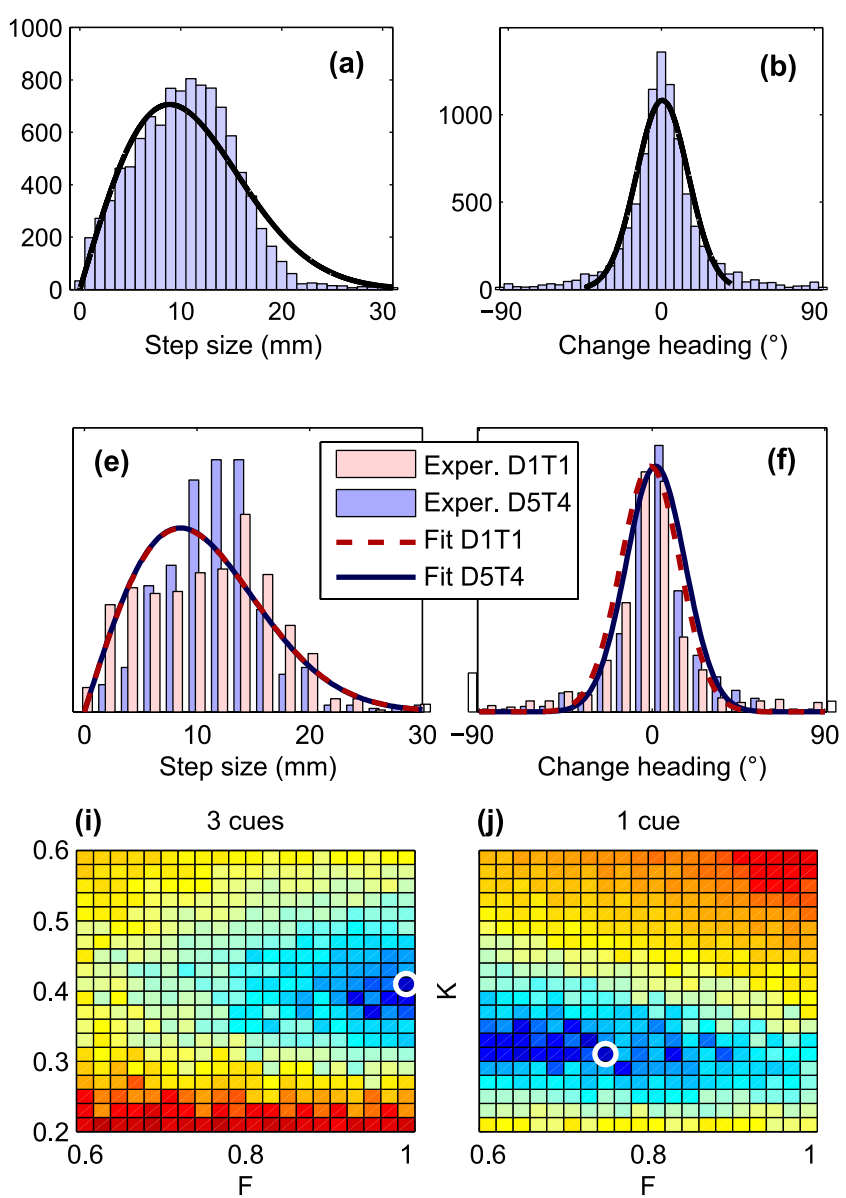

Fig. 2 Comparing data and model. (a)-(f) Visualisation of the random variables distributions: histograms show the data, solid lines the fitted probability density functions as used in the model. (a) Rayleigh distribution of the step size (b) normal distribution of the heading change (c) exponential distribution of the path length on the pool border (d) log-normal distribution of the angle at which rats leave the border (e), (f) Comparison of the distributions for untrained (day 1, trial 1) vs. trained rats (day 5 , trial 4). Fitted distributions show no significant difference. (g) Lag 1 (0.2 s) autocorrelation of the heading change over days. Markers indicate the mean over 8 rats and 4 trials, errorbars indicate the standard error of the mean (SEM). (h) Fit of the open loop model, i.e. identification of the parameters $A_{i}$. Solid:

latencies shows that this cue-based egocentric strategy is more efficient in the 3-cues case than in the 1-cue case (Fig. 3(b)). Although the escape latencies were significantly reduced compared to random swimming (50\% and $32 \%$ for 3 -cues and 1 -cue group, respectively), they did not reach the performance of fully trained rats. For example, Fig. 3(b) demonstrates that simulated animals in the 3-cues group reach escape latencies of $19 \mathrm{~s}$ whereas animals in the laboratory typically reach $10 \mathrm{~s}$ or less following 5 days of training (Kealy et al. 2008). At this point it is important to note that simulating the one cue experiment with the strong
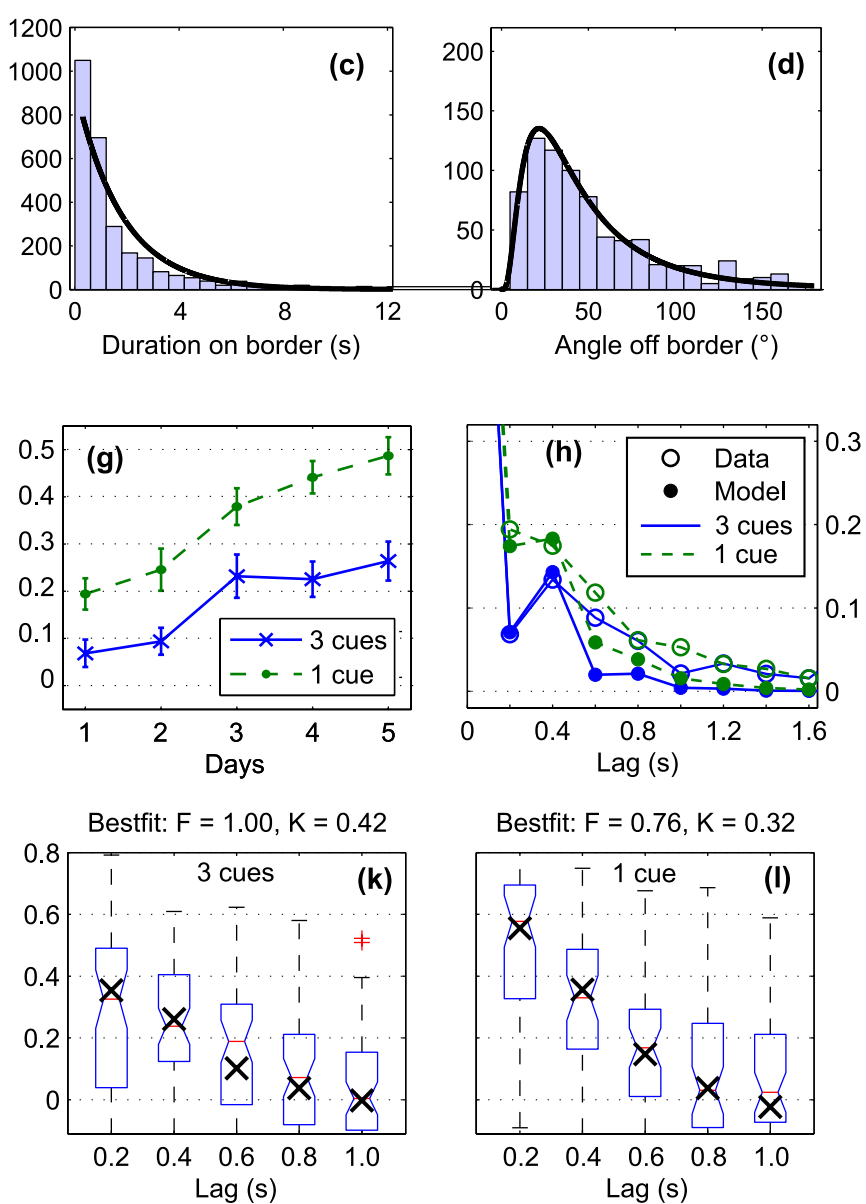

3 cues case. Dashed: 1 cue case. (i)-(I) Fit of the closed loop model, i.e. identification of the feedback parameters $\mathrm{K}$ and $\mathrm{F}$. (i), (j) Colour contour plot visualising the values of the costfunction (sum of squares error of the autocorrelation function) for different control parameters. Darker areas correspond to a better fit, the white circle indicates the best fit. (k), (I) Comparison of the autocorrelation function of the heading change of data and model. Box plots show the data, horizontal lines indicate the median, notches the $95 \%$ confidence interval, boxes the lower and upper quadrille, whiskers the extreme values and "+" outliers. The exmarks " $x$ " indicate the mean autocorrelation function of the model as obtained from $>500$ simulation runs

control parameters (as identified from the 3-cues data) increases the escape latencies significantly compared to the nominal control parameters (as identified from the 1-cue data) for cue usages of more than $60 \%$. This decrease of performance worsens the more the rat uses the cue (Fig. 3(b), red-dash-dotted line). A weaker control is therefore beneficial in a 1-cue scenario, explaining the difference of feedback strength identified in the previous section $\left(K_{3 \text {-cues }}>K_{1 \text {-cue }}\right)$.

A third simulation experiment, implemented an allocentric place navigation strategy, assuming the rats know the platform location with varying degrees of 


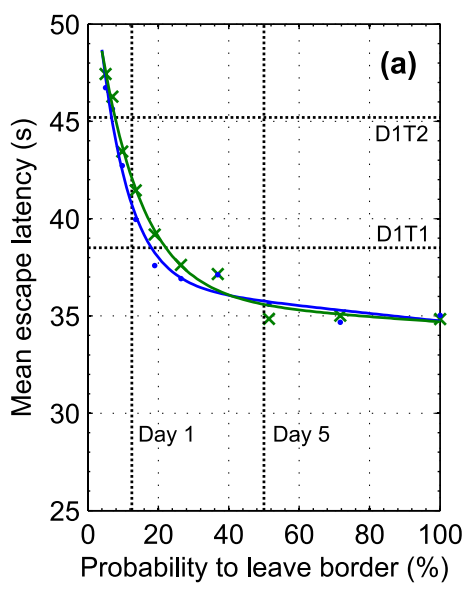

(d)

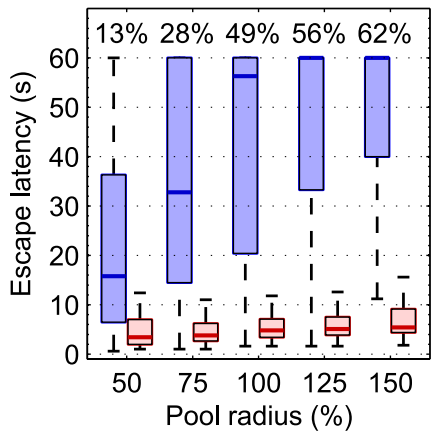

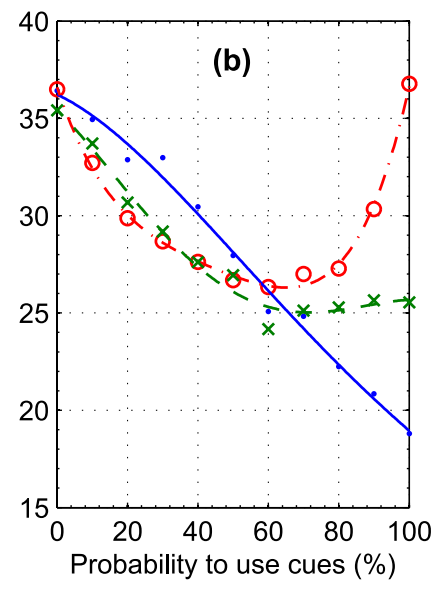

(e)

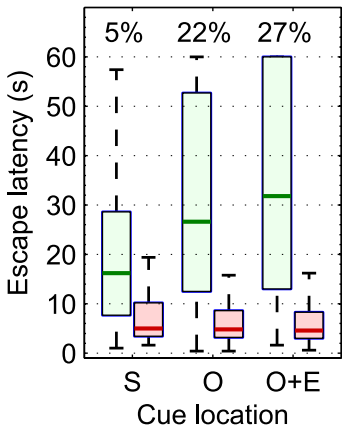

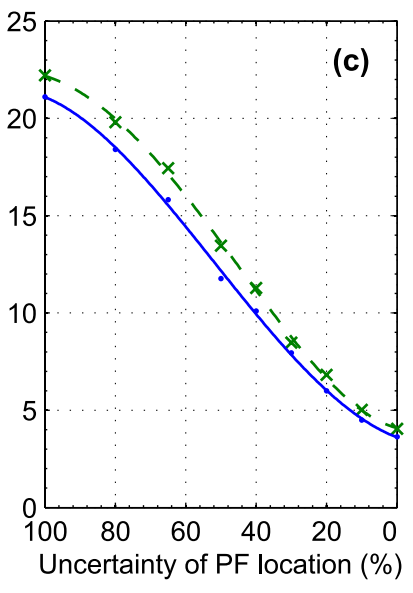

(a)

$$
\begin{aligned}
& \longrightarrow 3 \text { cues } \\
& -*-1 \text { cue }
\end{aligned}
$$

(b)

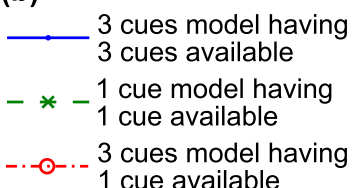

(c)

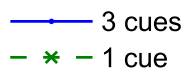

(f)

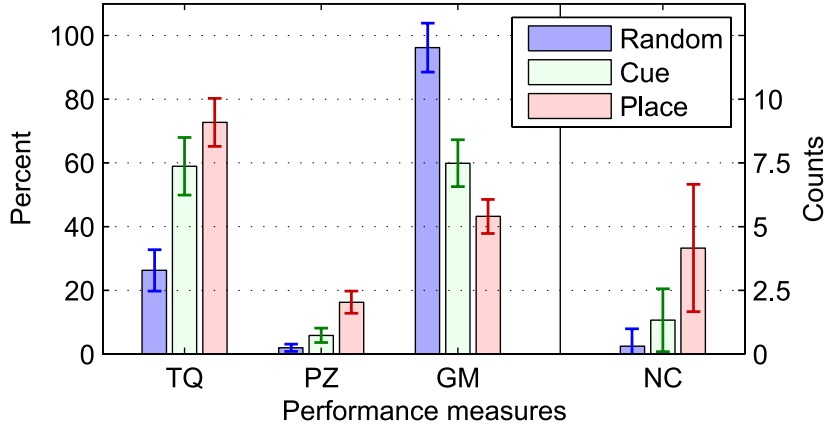

the percentage of unsuccessful trials. (d) Different pool sizes ( $\mathrm{x}$ axis). Blue, left-hand-side: random walk model (untrained rats of day one, i.e. probability to leave border $=1 / 8$ ). Red, right-handside: place control model (trained rats of day five, i.e. probability to leave border $=1 / 2$ ). (e) Different cue locations. Green, lefthand-side: egocentric cue-based strategy (3 cues, probability to use cues $=100 \%$ ); S: cues in same quadrant as platform, simulated rats approach cues $\left(\alpha_{\text {desired }}=\alpha_{\text {cues }}\right)$; O: cues in opposite quadrant as platform, simulated rats swim away from cues perfectly $\left(\alpha_{\text {desired }}=\alpha_{\text {cues }}+180^{\circ}\right) ; \mathrm{O}+\mathrm{E}$ : cues in opposite quadrant as platform, simulated rats swim away from cues but with an directional error $\left(\alpha_{\text {desired }}=\alpha_{\text {cues }}+180^{\circ}+\epsilon\right.$, where $\epsilon$ is normal distributed with zero mean and standard deviation $\left.10^{\circ}\right)$. Red, right-hand-side: place-control strategy (uncertainty $=30 \%$ ); S, O: rat swims to its assumed platform location directly $\left(\alpha_{\text {desired }}=\right.$ $\left.\alpha_{\mathrm{PF}, \text { rat }}\right), \mathrm{O}+\mathrm{E}$ : rat swimms to its assumed platform location with an directional error $\left(\alpha_{\text {desired }}=\alpha_{\mathrm{PF}}\right.$, rat $\left.+\epsilon\right)$. (f) Predictions of different performance measures for the random walk model (blue, left-hand-side bars), the egocentric cue-based strategy with 3 cues (green, middle boxes) and the place-control strategy (red, right-hand-side bars). In the simulations, trained rats are allowed to swim for $60 \mathrm{~s}$ without an escape platform. Errorbars indicate standard variations. $T Q$ time in quadrant, i.e. percentage of time the rat swims within the correct pool quadrant. $P Z$ Percentage zone, i.e percentage of time the rat swims within a circular zone around the correct location. (Zone covers $1 / 9$ of the total pool area.) $G M$ Gallagher measure or average distance (in percent, normalised to pool radius), i.e the mean distance to the correct location over the trial. $N C$ Number of crossings, i.e. the number of times the rat swims over the correct location 
uncertainty (similar to a cognitive map, Burgess 2008). These uncertainties were represented by two dimensional Gaussian distributions with varying degrees of standard deviation. The simulated rats' assumed platform location is the true platform location plus a random deviation drawn from the uncertainty distribution:

$x_{\mathrm{PF}, \text { rat }}=x_{\mathrm{PF}, \text { true }}+\rho \sin (\theta)$,
$y_{\mathrm{PF}, \text { rat }}=y_{\mathrm{PF}, \text { true }}+\rho \cos (\theta)$,

where $\rho$ is a normally distributed random number with zero mean and $\theta$ a uniformly distributed random number in the interval $[0,180)$. Once the simulated rat successfully navigated to its assumed platform location while realising there was no platform, it dropped that assumption and chose a new platform location, again by drawing from the uncertainty distribution. An analysis of the resulting escape latencies demonstrates that animals possessing little knowledge of the platform location (large uncertainty) can solve the task very effectively. Indeed, an uncertainty of about half the pool radius is sufficient to explain the escape latencies after 5 days of training (10 s, Fig. 3(c)). The situation of a perfectly learned platform location, i.e. with no uncertainty, results in very low escape latencies (4s). Such low escape latencies have been observed for over-trained rats (12 days of training, Kealy et al. 2008).

\subsection{Model predictions}

Water maze experiments depend on several factors: physical ones such as pool size and platform or cue location as well as behavioural ones. All are reflected as model parameters, which can easily be altered in simulations for generating model-based predictions. This is illustrated at the example of three types of experiments. First, changing the pool size has a major effect for untrained rats (random walk strategy), but not for trained rats (place-control strategy), see Fig. 3(d). Second, having the platform on the opposite side of the clues is more difficult with a cue-based strategy, but identical for a place navigation (Fig. 3(e)). Finally, simulated retention trials allow for comparison of different strategies and performance measures. For example, the Gallagher measure is best suited to distinguish the cuebased from the place-control strategy (no overlap of errorbars, Fig. 3(f)). Summarising, such model prediction are helpful in screening through possible experimental setups to uncover the most promising ones that should be performed in a real experiment.

\section{Discussion}

\subsection{The role of feedback control}

Developing a control system that robustly tracks a desired variable is a problem commonly faced by engineers. The standard solution is integral feedback control, in which the time integral of difference between actual value and desired value, is fed back into the system. A heating system controlled by a thermostat is one well-known example. Because temperature, which is proportional to the integral of heat (the output of the heater), is compared to the desired temperature and fed back into this closed-loop system, the difference between the room temperature and the desired temperature approaches zero despite external environmental disturbances or variations in the heater. Here, we have the same situation. The heading, which is the integral of the heading change, is fed back (Fig. 1(c)). Hereby, the role of the integrator is taken by the random walk model. It is therefore not necessary to use integral action within in the controller in order to achieve a zero tracking error in steady state.

The identified feedback parameters ( $K_{1 \text {-cue }}<K_{3 \text {-cues }}$, see Section 4) suggest that the more navigational cues are available, the more the animal seems confident, i.e. applies a stronger, more stringent control strategy. Our simulations showed the advantage of weaker, more moderate control in the one cue case as it allows for exploring a greater area.

\subsection{The role of the filter}

In engineering, a low pass filter is a simple but effective way to reduce (measurement) noise. Here we have a somewhat noisy situation in the one cue case where the rat's positional inference is impaired. It makes therefore sense that rats use a filter if only few or uncertain navigational cues are available $\left(F_{1 \text {-cue }}<F_{3 \text {-cues }}\right.$, see Section 4). A biological interpretation of this result is that animals navigating with more available cues rely on their immediate visual information, whereas animals with a limited number of available cues rely more on past information.

\subsection{Fitting the model into underlying neural circuits}

The neurophysiology of spatial memory and learning involves different brain regions depending on environment, experimental conditions and strategy employed by the rat (Fig. 1(b)). For example, the taxon (egocentric) pathway directly projects the visual cortex onto motor neurons in the striatum (Sheynikhovich et al. 
2009). In contrast, the locale (allocentric) pathway additionally involves hippocampal and parahippocampal regions and the brain's spatial representation system. In these (para) hippocampal regions, the rats location and head direction is represented by neurons called place cells and head direction cells, respectively. In the parahippocampus, grid cells of the entorhinal cortex are thought of incorporating the rats self movements (motor actions) in a process called path integration (Burgess 2008).

There is an extensive literature modelling the neuronal processes (e.g. Sheynikhovich et al. 2009; Burgess 2008) which ultimately cause the animal's movements. In contrast, the here proposed behavioural model focuses on the dynamics of the resulting movements. Models on both levels, the neuronal and the behavioural one, are necessary for a complete picture in which the loop can be closed via the environment (Fig. 1(b)). We suggest that the parameters of the dynamic model change depending on which neuronal pathway is activated. For example, the higher control gain $K$ in the 3-cue case might be linked to the synaptic projections from place cells to motor neurons in the nucleus accumbens in the ventral striatum (locale system). Further, the increased filter constant $F$ in the 1-cue case could be linked to increased activation of the projections from view cells to motor neurons in the caudate putamen in the dorsal striatum (taxon system, Sheynikhovich et al. 2009). However, the parameters $K$ and $F$ are probably not independent from each other and both are likely to be influenced by several, possibly overlapping neuronal processes.

\section{Conclusions}

Our modelling results concerning the 3-cues and 1cue group are in concordance with earlier reports. Harvey et al. (2009) revealed no significant difference between the groups in terms of the gross measures used (e.g escape latencies) demonstrating that both groups learned the task effectively, but also found different behavioural patterns. Here we provide a mathematical model explaining the group differences and revealing that both groups control their movements differently.
The parameters of the heading change model were identified in open loop and seem not to differ considerably. In contrast, the feedback parameters as identified in closed loop are clearly distinct. This demonstrates that the model can reveal inherent procedural differences not visible in gross measures. The model parameters are influenced by several factors that could be controlled in experiments (e.g. brain lesions, training schemes). This makes the model a useful tool for analysis of experiments for which swimming paths are recorded. The fitted model parameters can be understood as higher level measures reflecting behavioural and neurophysiological differences.

\section{References}

Burgess, N. (2008). Spatial cognition and the brain. Annals of the New York Academy of Sciences, 1124, 77-97. doi: 10.1196/annals.1440.002.

Cain, D. P., \& Saucier, D. (1996). The neuroscience of spatial navigation: Focus on behavior yields advances. Reviews of Neuroscience, 7(3), 215-231.

Harrison, F. E., Reiserer, R. S., Tomarken, A. J., \& McDonald, M. P. (2006). Spatial and nonspatial escape strategies in the Barnes maze. Learning and Memory, 13(6), 809-819. doi:10.1101/lm.334306.

Harvey, D. R., Brant, L., \& Commins, S. (2009). Differences in cue-dependent spatial navigation may be revealed by in-depth swimming analysis. Behavioural Processes, 82(2), 190-197. doi:10.1016/j.beproc.2009.06.008.

Kealy, J., Diviney, M., Kehoe, E., McGonagle, V., O'Shea, A., Harvey, D., et al. (2008). The effects of overtraining in the Morris water maze on allocentric and egocentric learning strategies in rats. Behavioural Brain Research, 192(2), 259263. doi:10.1016/j.bbr.2008.04.009.

Maei, H. R., Zaslavsky, K., Teixeira, C. M., \& Frankland, P. W. (2009). What is the most sensitive measure of water maze probe test performance? Frontiers in Integrative Neuroscience, 3, 4. doi:10.3389/neuro.07.004.2009.

Moghaddam, M., \& Bures, J. (1996). Contribution of egocentric spatial memory to place navigation of rats in the Morris water maze. Behavioural Brain Research, 78(2), 121-129. doi:10.1016/0166-4328(95)00240-5.

Morris, R. G., Garrud, P., Rawlins, J. N., \& O'Keefe, J. (1982). Place navigation impaired in rats with hippocampal lesions. Nature, 297(5868), 681-683.

Sheynikhovich, D., Chavarriaga, R., Strösslin, T., Arleo, A., \& Gerstner, W. (2009) Is there a geometric module for spatial orientation? Insights from a rodent navigation model. Psychology Review, 116(3), 540-566. doi:10.1037/a0016170. 